\title{
Medición de la usabilidad del diseño de interfaz de usuario con el método de evaluación heurística: dos casos de estudio
}

\section{Measuring the usability of the design of user interface with the Heuristic Evaluation Method: two case studies}

\author{
Guadalupe García Toribio $^{1}$ (D) , Yesenia Polvo Saldaña $^{1}$ (D) José Juan Hernández Mora ${ }^{1}$ \\ , María Janaí Sánchez Hernández ${ }^{1}$ (D) , Higinio Nava Bautista ${ }^{1}$ (D) , César Alberto Collazos \\ Ordóñez $z^{2}$, Julio Ariel Hurtado Alegría ${ }^{2}$
}

${ }^{1}$ División de Estudios de Posgrado e Investigación, Tecnológico Nacional de México. Instituto Tecnológico de Apizaco, Apizaco, Tlaxcala, México.

${ }^{2}$ Facultad de Ingeniería Electrónica y Telecomunicaciones, Departamento de Sistemas, Universidad del Cauca, Popayán, Colombia.

guadalupe.garcia.toribio@gmail.com, yeseniapolvos@gmail.com,juan.hm@apizaco.tecnm.mx,janai.sh@ apizaco.tecnm.mx, higinio.nb@apizaco.tecnm.mx, ccollazo@unicauca.edu.co, ahurtado@unicauca.edu.co

(Recibido: 27 noviembre 2018; aceptado: 4 febrero 2019)

\begin{abstract}
Resumen
La usabilidad es un factor determinante que actualmente está cobrando mayor relevancia, provocando que las empresas de software aumenten el nivel de calidad en sus fases de desarrollo. Una fase por considerar es el diseño de la interfaz de usuario, donde se debe valorar la usabilidad como un atributo de calidad, permitiendo una interacción sencilla, agradable e intuitiva, además de discutir los objetivos y requerimientos. Se visualiza que algunos sistemas no cuentan con la práctica de evaluación, provocando conflicto en identificar errores, mantenimiento y rediseño, por lo que es necesario el uso de una Evaluación Heurística (EH), que permita comprobar el nivel de usabilidad. En este trabajo se describen dos evaluaciones de un Sistema Académico - Administrativo, utilizando el método EH de Nielsen para evaluar el diseño de los módulos en su atributo de calidad con base en la usabilidad, presentando los resultados de las evaluaciones.
\end{abstract}

Palabras clave: Atributos de calidad, Usabilidad, Evaluación heurística, Experiencia de usuario.

\begin{abstract}
Usability is a key factor that is currently getting greater importance, leading software enterprises to increase the level of quality in their development stages. A phase to consider is the interface design, where it is due to value usability as a quality attribute, allowing a simple and intuitive interaction, as well to consider objectives and requirements. Is displayed that some systems don't consider the evaluation practice, causing conflict identifying errors, maintenance, redesign, therefore its necessary the use of a Heuristic Evaluation (HE), allowing check the usability level. In this paper we describe two evaluations of an Academic- Administrative System, using the method HE by Nielsen to evaluate the modules design in their quality attribute based on usability, presenting the evaluations results.
\end{abstract}

Keywords: Quality attributes, Usability, Heuristic evaluation, User experience. 


\section{Introducción}

Actualmente con el avance tecnológico universal los requerimientos de los usuarios son cada vez más complejos, por lo cual se hace uso de nuevas tácticas a implementar, para que personas de todas las edades, capacidades y diferentes perfiles socioculturales, puedan interactuar de forma eficiente, cómoda e intuitiva con cualquier sistema, producto o aplicación de software, por lo que se hace pertinente recurrir a la medición de usabilidad. El estándar ISO 9241 la define como "la extensión en que un producto puede ser utilizado por usuarios especificos para alcanzar objetivos determinados con efectividad, eficiencia y satisfacción dentro de un contexto" (International Organization for Standardization, 1998, p.2). Este término solo ha sido utilizado para analizar aquellos factores que contribuyen a que un sistema web resulte fácil de utilizar, ya que la creciente demanda de productos de software ha hecho que la usabilidad sea inherente y, por lo tanto, es de suma importancia que garantice que el producto final sea de calidad y se cumplan los objetivos definidos desde la creación de este. Jakob Nielsen define "la facilidad de uso como la calidad y atributo en relación con algo tan fácil de usar" (Chimarro Chipantiza, Mazón Olivo \& Cartuche Calva, 2015, p.15).

Debido a la implementación incorrecta de la usabilidad, se puede llegar a obtener un sistema deficiente con una mala estructuración de elementos relacionados o en un caso crítico al fracaso total. Por tal motivo existen distintos métodos y escenarios de evaluación para medir la usabilidad de un sistema, tras analizarlos y comprenderlos se determinó utilizar el método de Evaluación Heurística de Nielsen (1995). Esta consiste en un grupo de expertos que valoran si los elementos de la interfaz del usuario de un sistema web o aplicación corresponden con los principios de usabilidad establecidos a partir de una lista de heurísticas. La técnica consigue detectar un gran número de problemas de usabilidad en el diseño de un sistema web, provocando un menor coste que las técnicas que involucran usuarios (Montes de Oca, 2004).

Por lo cual, es importante implementar una evaluación en el diseño del sistema, para poder explorar y clasificar los problemas encontrados en esta etapa y así poder solucionarlos evitando pérdida de tiempo, costo y trabajo, entre otros factores.

Suárez Torrente (2011) menciona que "la evaluación de la usabilidad es una de las tareas más importantes que deben emprenderse cuando se desarrolla una interfaz de usuario", por lo que evaluar este atributo constituye solo una parte de la ingeniería de la usabilidad.

El presente documento aborda la evaluación de usabilidad en dos diseños de interfaz de un Sistema Académico - Administrativo, con dos módulos diferentes denominados "Residencias Profesionales" y "Tutorías", los cuales fueron creados sin seguir métricas de usabilidad, con el fin de mitigar riesgos de codificación e interfaces de usuario posteriores, así como tomar algunas acciones correctivas sobre la estructura, por lo cual se reporta una experiencia de evaluación en el diseño.

Este artículo está organizado de la siguiente forma: en la sección 2 describe los diferentes métodos de evaluación de usabilidad; en la sección 3 se describe el ajuste del método de Evaluación Heurística de Nielsen; en la sección 4 se presenta el reporte de experiencia que incluye cómo fue preparada, conducida, sistematizada y, en adición, los resultados obtenidos con la experiencia de evaluación en los diseños de los módulos de "Residencias Profesionales" y "Tutorías" con el método de EH de Nielsen, donde se midió el diseño del prototipo de interfaz con foco de atributo de usabilidad; finalmente, en la sección 5 se presentan las conclusiones.

\section{Tipos de métodos de evaluación de usabilidad}

Los métodos tradicionales de evaluación de usabilidad para sistemas Web son cuestionados actualmente por no tener en cuenta al usuario (Abran, Khelifi, Suryn \& Seffah, 2003), en relación con la experiencia obtenida en la interacción con el sistema. Preece, Rogers \& Sharp (2005) definen a un sistema como "un proceso sistemático de recolección de datos con la finalidad que el usuario utilice el artefacto para realizar sus tareas en el entorno computacional". En este sentido, se ha tenido la necesidad de diseñar un prototipo de sistema Web, que integre la usabilidad como una parte importante del sistema, ya que es el atributo de calidad que determina si el manejo del sistema es fácilmente aprendido, difícilmente olvidado, no provoca errores, satisface a sus usuarios, y si eficientemente resuelve las tareas para las que fue proyectado. Los métodos de evaluación de usabilidad varían según las características del sistema o la etapa de desarrollo (Bevan, 1991), entre otros aspectos, que se dividen en tres categorías que reflejan una amplia variedad de métodos de evaluación. Algunos de ellos se mencionan a continuación: 


\subsection{Inspección}

\section{Evaluación Heurística}

Es un método que tiene como finalidad analizar la conformidad de la interfaz con principios reconocidos mediante la inspección de varios evaluadores. Estos evaluadores se basan en principios previamente definidos. Se utiliza esta evaluación en la usabilidad para medir la calidad de la interfaz de un sistema en relación con la posibilidad de ser aprendido y usado por un grupo de usuarios en un contexto de uso (Manzari \& Trinidad-Christensen, 2006).

Este método se destaca debido a su coste bajo, no obstante, este mismo puede variar dependiendo de cuántos evaluadores estén contemplados para el mismo. Sin embargo, el uso de EH trae resultados positivos. Según Nielsen, en este método se detecta, aproximadamente, el $42 \%$ de los problemas graves de diseño, así como un 32\% de problemas menores; todo esto depende de la cantidad de personas que fungen como evaluadores (Nielsen, 1994c).

\section{Recorrido cognitivo}

El enfoque de este método es evaluar la facilidad de un diseño al ser entendido; sus evaluadores construyen diversos escenarios y tareas, para posteriormente asumir el rol del usuario para trabajar con la interfaz (Blackmon \& Bainbridge, 2004). Su principal idea es identificar los objetivos de los usuarios y los problemas que tendrían en la interacción. La ventaja del recorrido cognitivo es que está sustentado en el aprendizaje basado en la exploración y solo requiere del criterio de un experto. Wharton et al. (1994) menciona que "esta técnica se debe centrar en la evaluación de la facilidad de aprendizaje".

\subsection{Indagación}

\section{Entrevista}

Se toma este instrumento con el fin de obtener información sobre la percepción de los usuarios acerca de distintos aspectos del sistema. Adicional a las entrevistas, también se crean cuestionarios; estos ocasionan que la entrevista sea menos flexible, pero hace que lleguen a más usuarios y a un panorama más amplio, para generar reportes estadísticos con mayor facilidad. Estos medios implican también un coste bajo al momento de realizar la evaluación ya que resulta de gran ayuda a los recursos tecnológicos (Claros Gómez, 2006).

\section{Observación de campo}

La característica de este método es prestar atención en los entornos de ejecución de los usuarios representativos, donde se diseñan entrevistas y sesiones de observación directa al entorno, ya que su objetivo principal es comprender cómo son realizados los diversos procesos y tareas, además de saber y entender su modelo mental (Nielsen, 1994b).

\subsection{Test}

\section{Interacción constructiva}

Se entiende como una derivación del método "pensando en voz alta", el cual Nielsen describe como el medio en el que se pueden expresar múltiples pensamientos, sentimientos, puntos de vista y opiniones de cómo luce el aspecto, funcionalidad, aprendizaje, entre otros, mientras se va interactuando con el sistema. El método de interacción constructiva implica tener en vez de uno, dos usuarios que hagan el test al sistema conjuntamente. Estas dos personas intercambian opiniones y dan como resultado el grado de usabilidad en el sistema (Reyes Vera \& Libreros Giraldo, 2011).

\section{Método Conductor}

Este tipo se cataloga en los diferentes usuarios ya que se centra en los usuarios inexpertos. Su objetivo principal es averiguar las necesidades de información de los involucrados evaluados, de tal manera que 
se proporcione un mejor entendimiento y documentación de un posible rediseño de la interfaz, para así aprovechar el tiempo de recopilación (Lorés \& Granollers, 2004).

Aunado a los anteriores tipos de evaluaciones de usabilidad estudiados, se descubrió que en la categoría de indagación la "entrevista y observación de campo" se enfocan más en la visualización del usuario que en su entorno de trabajo para la realización de la evaluación, ya que consiste en hablar con los usuarios, observarlos detenidamente usando el sistema en trabajo real y obtener respuestas a preguntas formuladas verbalmente o por escrito. En el caso de la categoría de test la "interacción constructiva y el método conductor" son evaluaciones que se enfocan en el proceso de interacción en el sistema entre uno o más usuarios inexpertos, para poder intercambiar ideas y rediseño del mismo, ocasionando que la evaluación no sea la esperada.

En este sentido, fue seleccionado el EH de Nilsen como método de evaluación de la usabilidad por inspección, en el cual se muestra una lista de heurísticas referentes a evaluar, presentando como característica del método la resolución y hallazgo de errores de usabilidad, los cuales son en gran medida elementos que se han definido para efectuar la inspección de la interfaz. También se describe el tratamiento del contenido que debe contener la información necesaria acorde a la realidad social, además de que toma en cuenta la interacción entre el usuario y el diseño con un grado de atracción en la complacencia de los resultados.

\section{Ajuste del método de Evaluación Heurística de Nielsen}

Dado el objetivo de la evaluación en los dos casos de estudio, se utilizaron los principios de Heurística de Nielsen, con los cuales se estipula si el sistema es usable o no. Cabe resaltar que estas son algunas reglas que definen un concepto de evaluación (Nielsen, 1994a). Para dar paso a la EH se definieron los principios a realizar basados en el estudio de diseño del prototipo, partiendo de una lista de elementos tomados en cuenta, los cuales son presentados a continuación:

I. Visibilidad del diseño del prototipo del sistema. Es indispensable establecer que cada diseño del prototipo debe informar al usuario final dónde se encuentra, qué está pasando y qué tarea está realizando en todo momento. Es importante mostrar que todos y cada uno de los enlaces que se exploran están claramente señalados para permitir al usuario conocer cómo interactuar con el sistema.

II. Relación entre diseño y el mundo real. Como función primordial el diseño debe utilizar el lenguaje de los usuarios y no uno propio relacionado con la tecnología, además de ser claro y comprensible. Se deben utilizar conceptos que sean entendibles, así como palabras con un significado conocido. Por último, al incluir íconos, imágenes o gráficos, deben generar un significado representativo para determinar una mejor interacción en el sistema, evitando equivocaciones.

III. Navegación y libertad en el diseño del prototipo. Para tener una mejor interacción el usuario debe tener la sensación de que posee el control de navegación del diseño, dándole la opción de realizar la acción de regresar al punto anterior con la ayuda de botones, con la finalidad de ofrecer una buena navegación y desplazamiento entre módulos y/o pantallas.

$I V$. Consistencia y estándares. En cada diseño de navegación realizada debe existir coherencia entre sintaxis, lenguaje, apariencia visual, iconos y efectos de nombres de un enlace, así como el sitio al que apunta. A su vez, todos y cada uno de ellos debe tener contenido, sin excepción, con la finalidad de que sea llevado a un sitio en particular.

V. Prevención de errores. Se presentan mensajes que prevengan posibles errores para una mejor interacción, evitando que el problema ocurra. Es conveniente hacer un diseño sin inducción a cometer errores. Para alcanzar este objetivo se crean sugerencias de interacción, ya sea formato de texto, moneda, fechas, teléfonos, asistentes de búsqueda, entre otros. Si ha sucedido el error se debe informar al usuario qué ha ocurrido, ayudándolo a encontrar la solución a su inconveniente.

VI. Reconocer en lugar de recordar. El principal objetivo es que cada recurso implementado sea fácilmente reconocible, que cada enlace a acceder pueda ser identificado claramente y permita reconocer dónde se encuentra el usuario en todo momento. Con esto no tendrá que recordar la información de una parte del diálogo a otra. Aquí recaen dos conceptos, reconocimiento y recuerdo, siendo más fácil reconocer que recordar; tomando como resultado que el reconocimiento es práctico, rápido y sin complejos.

VII. Estética y diseño minimalista. La información presentada al usuario debe ser relevante, favoreciendo el reconocimiento antes del recuerdo, mostrando en cada diseño la correcta clasificación, organización y distribución del diseño del prototipo. 
VIII. Reconocimiento, diagnóstico de errores. Todo diseño es propenso a contener errores. En algunas ocasiones es difícil reconocerlos, por lo que se hace necesario la utilización de mecanismos para solucionarlos, los cuales auxilian al usuario para una mejor interacción. Todo error mostrado debe ser explícito, entendible, preciso y constructivo. También, estos reconocimientos permiten, en cierta forma, educar al usuario para ofrecer mejor uso.

IX. Tratamiento del contenido. El contenido debe ser adecuado con la realidad social y cultural del usuario. Esto constituye un valor agregado con relación al mismo en otro medio.

$X$. Pedagogía. En un prototipo es fundamental la definición de perfiles de usuarios del diseño de la interfaz a elaborar, ya que determina el objetivo de la discriminación de cada sección a interactuar.

XI. Satisfacción. La diversa interacción entre el usuario y el diseño debe contener un grado de atracción en la complacencia de los resultados, además de una potenciación de participación del usuario en las tareas del sistema.

\section{Reporte de experiencia: evaluación y resultados de usabilidad en los módulos del sistema académico - administrativo con el método de EH de Nielsen}

En esta sección se presenta el proceso propuesto con respecto a la adaptación del EH de Nielsen (1994a) mostrando la ejecución de la evaluación de acuerdo con el objetivo establecido, para evaluar la usabilidad del diseño de los módulos "Residencias Profesionales" y "Tutorías" del Sistema de Servicios Integrales de una Institución de Educación Superior, al tiempo se analizan sus diversos factores y atributos de calidad. Abordando el plan establecido que se describe en la Tabla 1 se observan los valores de medición que serán otorgados para cada heurística. Los principios de evaluación son presentados de la Tabla 2 a la 12. En cada principio heurístico, se listan algunos de los puntos particulares para ser cumplidos en la evaluación a través de este método.

Tabla 1. Mediciones que dan valor a los heurísticos

\begin{tabular}{lll} 
Valor & \multicolumn{1}{c}{ Medición } & \multicolumn{1}{c}{ Observaciones } \\
\hline 1 & Muy en desacuerdo & Lo evaluado no realiza la actividad o no muestra el contenido que ofrece. \\
\hline 2 & En desacuerdo & $\begin{array}{l}\text { Lo evaluado desarrolla la actividad o muestra un contenido, pero en general, lo } \\
\text { recibido por el evaluador no aporta a la experiencia general del sistema. }\end{array}$ \\
\hline 3 & Neutro & $\begin{array}{l}\text { Lo evaluado desarrolla la actividad o muestra un contenido relativamente útil, } \\
\text { pero podría ser mejor. }\end{array}$ \\
\hline 5 & De acuerdo & Lo evaluado desarrolla la actividad o muestra un contenido útil. \\
\hline 5 & Muy de acuerdo & $\begin{array}{l}\text { Lo evaluado desarrolla la actividad o muestra un contenido útil que cumple o } \\
\text { excede la expectativa del usuario. }\end{array}$ \\
\hline
\end{tabular}

Nota: tomada de Sánchez I (2000).

Tabla 2. Checklist para visibilidad del diseño del prototipo del sistema

$\begin{aligned} & \text { I. Visibilidad del diseño del prototipo del sistema } \\ & 1.1 \text { El diseño muestra claramente dónde se encuentra el }\end{aligned}$
usuario.

Nota: tomada de Sánchez I (2000).

Tabla 3. Checklist para relación entre diseño y mundo real

\begin{tabular}{l} 
II. Relación entre diseño y mundo real \\
2.1 El lenguaje es claro. \\
\hline 2.2 Los conceptos utilizados son entendibles. \\
\hline 2.3 Las palabras son de significado conocido. \\
\hline 2.4 Los iconos generan significado.
\end{tabular}

Nota: tomada de Sánchez I (2000). 
Tabla 4. Checklist para navegación y libertad en el diseño del prototipo

\section{$\begin{array}{llllllllll}\text { III. Navegación y libertad en el diseño del prototipo } & 1 & 2 & 3 & 4 & 5 & \text { Observaciones }\end{array}$}

3.1 Es fácil regresar al punto inmediatamente anterior.

3.2 Es fácil volver a la página principal desde cualquier página.

3.3 Provee botones propios para volver o dar paso a otra página.

Nota: tomada de Sánchez I (2000).

Tabla 5. Checklist para consistencia y estándares

$\begin{array}{lllllll}\text { IV. Consistencia y estándares } & 1 & 2 & 3 & 4 & 5 & \text { Observaciones }\end{array}$

4.1 Existe coherencia entre el nombre de un enlace y el sitio al que apunta.

4.2 Todos los enlaces tienen contenido.

4.3 Existen coherencias entre el título de una página y su contenido.

4.4 Sólo existe un botón o enlace que lo lleve a un mismo sitio.

Nota: tomada de Sánchez I (2000).

Tabla 6. Checklist para prevención de errores

\begin{tabular}{|c|c|c|c|c|c|c|}
\hline V. Prevención de errores & 1 & 2 & 3 & 4 & 5 & Observaciones \\
\hline
\end{tabular}

5.1 Existen mensajes que prevengan posibles errores.

5.2 Es posible prever posibles errores.

5.3 El diseño del sistema no induce a cometer errores.

Nota: tomada de Sánchez I (2000).

Tabla 7. Checklist para reconocer en lugar de recordar

$\begin{array}{lllllll}\text { VI. Reconocer en lugar de recordar } & 1 & 2 & 3 & 4 & 5 & \text { Observaciones }\end{array}$

6.1 Los iconos son fácilmente reconocibles.

6.2 Los enlaces pueden identificarse claramente.

6.3 Es posible reconocer dónde se encuentra el usuario.

Nota: tomada de Sánchez I (2000).

Tabla 8. Checklist para estética y diseño minimalista

\begin{tabular}{lllllll} 
VII. Estética y diseño minimalista & 1 & $\mathbf{2}$ & $\mathbf{3}$ & $\mathbf{4}$ & $\mathbf{5}$ & Observaciones \\
7.1 La información es relevante. & & & & & & \\
\hline 7.2 El contenido está bien clasificado. & & & \\
\hline 7.3 El contenido está correctamente organizado. & & \\
\hline 7.4 El contenido está bien distribuido en el diseño.
\end{tabular}

Nota: tomada de Sánche z I (2000). 
Tabla 9. Checklist para reconocimiento, diagnóstico de errores

\begin{tabular}{lllllll} 
VIII. Reconocimiento, diagnóstico de errores & $\mathbf{1}$ & $\mathbf{2}$ & $\mathbf{3}$ & $\mathbf{4}$ & $\mathbf{5}$ & Observaciones \\
8.1 Es fácil reconocer cuando ocurre un error. & & & & & \\
\hline 8.2 Después que ocurre un error es fácil volver al sitio de origen. \\
\hline 8.3 Cuando ocurre un error existen mecanismos para solucionarlos.
\end{tabular}

Nota: tomada de Sánchez I (2000).

Tabla 10. Checklist para tratamiento del contenido

IX. Tratamiento del contenido
$\begin{aligned} & \text { 9.1 El contenido se adecua a la realidad social y cultural del } \\ & \text { usuario. }\end{aligned}$
$\begin{aligned} & \text { 9.2 El contenido del diseño constituye un valor agregado en } \\ & \text { relación al mismo contenido en otro medio. }\end{aligned}$
$\begin{aligned} & \text { 9.3 Existe opción de realizar consultas al autor o al administrador } \\ & \text { del sitio. }\end{aligned}$
$\begin{aligned} & \text { 9.4 Es posible ampliar la información accediendo a punteros } \\ & \text { relacionados con el tema. }\end{aligned}$

Nota: tomada de Sánchez I (2000).

Tabla 11. Checklist para pedagogía

\begin{tabular}{|c|c|c|c|c|}
\hline X. Pedagogía & 12 & 3 & 5 & Observaciones \\
\hline
\end{tabular}

Nota: tomada de Sánchez I (2000).

Tabla 12. Checklist para satisfacción

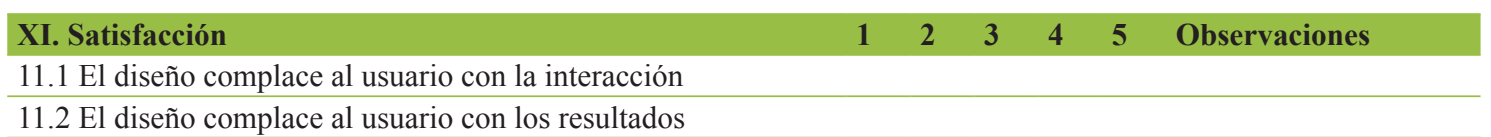

Nota: tomada de Sánchez I (2000).

Acorde a los principios identificados anteriormente se presentan los reportes de experiencia y se ejecutan los siguientes puntos en cada caso de estudio:

\subsection{Caso de estudio: Residencias Profesionales (RP)}

Identificación de los interesados que interactúan en el módulo (RP)

En el proceso de evaluación se definieron los perfiles y acciones de los usuarios que intervienen en el módulo, los cuales se muestran en la Tabla 13.

\section{Aplicación y resultados de la evaluación a un perfil}

En la evaluación del módulo de "Residencias Profesionales" se tomó una muestra de quince personas del perfil Residente. En la Tabla 14 son presentados los resultados Heurísticos obtenidos de cinco de las muestras, allí se visualizan los totales de cada Heurística y el total de cada Evaluación. Al analizar la puntuación otorgada por cada residente en cada heurística, se presentan similitudes en algunos apartados donde se puede determinar la falta o buena implementación de usabilidad, por lo que se estableció seguir 
con más evaluaciones para tener un mejor resultado de usabilidad. De igual manera los formatos utilizados para las evaluaciones se aplicaron para todos los perfiles que se mencionan en la Tabla 13.

Tras los resultados obtenidos anteriormente, se elaboró una tabla de medición que otorga valor al total de cada principio heurístico y al porcentaje total de la evaluación, obteniendo como resultado el nivel de usabilidad del diseño (ver Tabla 15). Con respecto a los resultados detallados de la Tabla 14 y la utilización de los valores de medición mencionados previamente, se presenta en la Tabla 16 el porcentaje total obtenido de la muestra de cinco evaluaciones al perfil Residente, dando como resultado tres evaluaciones con valor de "Muy bueno" y dos con valor de "Bueno", lo que demuestra que el diseño contiene atributos de usabilidad. Con los resultados obtenidos se realizó un análisis a cada una de las heurísticas, ya que en algunas de ellas se visualizó la falta de usabilidad, en las cuales se propusieron mejoras para un mejor diseño.

\section{Obtención de resultados generales}

En la Tabla 17 se visualiza el número de evaluadores de cada perfil, mostrando los porcentajes finales obtenidos. Con la utilización de la Tabla 15 del apartado (Porcentaje Total) se puede identificar el nivel de Usabilidad obtenido por cada Evaluación.

Tabla 13. Listado de perfiles que intervienen en el módulo de "Residencias Profesionales"

\begin{tabular}{|c|c|}
\hline Perfil & Acciones \\
\hline Residente & $\begin{array}{l}\text { Visualiza la convocatoria de residencias profesionales. } \\
\text { Visualiza banco de proyectos. } \\
\text { Selecciona proyecto de las diversas opciones. } \\
\text { Realiza pre-registro. } \\
\text { Descarga solicitud y reporte técnico para realizar residencias profesionales. } \\
\text { Solicita y entrega documentos requeridos para realización de residencias profesionales. } \\
\text { Recibe y entrega carta de presentación y aceptación a la empresa. } \\
\text { Descarga cronograma de actividades para su seguimiento. } \\
\text { Descarga seguimiento de residencia de evaluación dos veces durante todo el proceso } \\
\text { para su evaluación. } \\
\text { Descarga formato de reporte final para su realización y evaluación. } \\
\text { Descarga formato de evaluación final. } \\
\text { Entrega carta de liberación por parte de la empresa. }\end{array}$ \\
\hline Coordinador & $\begin{array}{l}\text { Elabora banco de proyectos. } \\
\text { Verifica datos del residente. } \\
\text { Elabora expediente del residente. } \\
\text { Asigna proyecto al residente. } \\
\text { Recibe todos los documentos de residencias del residente. } \\
\text { Elabora carta de liberación de residencia profesional. }\end{array}$ \\
\hline $\begin{array}{l}\text { Jefe del Departamento } \\
\text { Académico }\end{array}$ & $\begin{array}{l}\text { Asigna al revisor del dictamen y entrega oficio de asignación. } \\
\text { Autoriza el proceso de Residencia Profesional. } \\
\text { Asigna asesores internos y entrega oficio de asignación. } \\
\text { Asigna revisores y entrega oficio de asignación. }\end{array}$ \\
\hline $\begin{array}{l}\text { Integrante de Academia } \\
\text { (Revisor de Dictamen) }\end{array}$ & Provee el dictamen del proyecto. \\
\hline Asesor Interno & $\begin{array}{l}\text { Determina la cantidad de estudiantes y el perfil académico requerido para el desarrollo } \\
\text { del proyecto. } \\
\text { Asesora el residente durante todo el proceso de Residencia Profesional. } \\
\text { Firma cronograma de actividades. } \\
\text { Evalúa el seguimiento del residente. } \\
\text { Evalúa el reporte final del residente. } \\
\text { Evalúa formato final de Residencia Profesional. } \\
\text { Asigna calificación. } \\
\text { Firma portada de reporte final. }\end{array}$ \\
\hline $\begin{array}{l}\text { Gestión Tecnológica y } \\
\text { Vinculación (G.T. y V.) }\end{array}$ & $\begin{array}{l}\text { Identifica necesidades en las empresas, organismos o dependencias para generar } \\
\text { proyectos pertinentes a la Residencia Profesional. } \\
\text { Realiza carta de presentación. }\end{array}$ \\
\hline Servicios Escolares (S.E) & Responsable de emitir el acta de calificación de Residencia Profesional. \\
\hline
\end{tabular}


Tabla 14. Resultados detallados de cinco de las muestras de Evaluación Heurística al perfil Residente

\begin{tabular}{|c|c|c|c|c|c|c|}
\hline \multirow[t]{2}{*}{ Heurísticas } & \multirow[t]{2}{*}{ Preguntas } & \multicolumn{5}{|c|}{ Residentes } \\
\hline & & 1 & 2 & 3 & 4 & 5 \\
\hline \multirow[t]{3}{*}{ I. } & 1.1 & 5 & 5 & 4 & 5 & 5 \\
\hline & 1.2 & 4 & 4 & 5 & 4 & 5 \\
\hline & Total & 9 & 9 & 9 & 9 & 10 \\
\hline \multirow[t]{5}{*}{ II. } & 2.1 & 4 & 5 & 5 & 4 & 5 \\
\hline & 2.2 & 5 & 5 & 5 & 5 & 5 \\
\hline & 2.3 & 5 & 5 & 5 & 5 & 5 \\
\hline & 2.4 & 5 & 5 & 5 & 4 & 4 \\
\hline & Total & 19 & 20 & 20 & 18 & 19 \\
\hline \multirow[t]{4}{*}{ III. } & 3.1 & 5 & 5 & 5 & 5 & 5 \\
\hline & 3.2 & 4 & 3 & 4 & 3 & 4 \\
\hline & 3.3 & 4 & 3 & 5 & 5 & 5 \\
\hline & Total & 13 & 11 & 14 & 13 & 14 \\
\hline \multirow[t]{5}{*}{ IV. } & 4.1 & 5 & 4 & 4 & 5 & 4 \\
\hline & 4.2 & 5 & 5 & 5 & 4 & 5 \\
\hline & 4.3 & 4 & 4 & 3 & 4 & 5 \\
\hline & 4.4 & 3 & 4 & 3 & 5 & 4 \\
\hline & Total & 17 & 17 & 15 & 18 & 18 \\
\hline \multirow[t]{4}{*}{ V. } & 5.1 & 4 & 4 & 3 & 4 & 5 \\
\hline & 5.2 & 5 & 4 & 5 & 5 & 5 \\
\hline & 5.3 & 4 & 3 & 4 & 3 & 3 \\
\hline & Total & 13 & 11 & 12 & 12 & 13 \\
\hline \multirow[t]{4}{*}{ VI. } & 6.1 & 5 & 5 & 5 & 5 & 5 \\
\hline & 6.2 & 5 & 4 & 5 & 4 & 4 \\
\hline & 6.3 & 4 & 3 & 4 & 3 & 4 \\
\hline & Total & 14 & 12 & 14 & 12 & 13 \\
\hline \multirow[t]{5}{*}{ VII. } & 7.1 & 4 & 5 & 4 & 4 & 5 \\
\hline & 7.2 & 3 & 5 & 5 & 3 & 3 \\
\hline & 7.3 & 4 & 3 & 3 & 4 & 3 \\
\hline & 7.4 & 4 & 4 & 3 & 4 & 5 \\
\hline & Total & 15 & 17 & 15 & 15 & 16 \\
\hline \multirow[t]{4}{*}{ VIII. } & 8.1 & 3 & 3 & 3 & 4 & 4 \\
\hline & 8.2 & 4 & 3 & 4 & 3 & 3 \\
\hline & 8.3 & 4 & 4 & 4 & 3 & 3 \\
\hline & Total & 11 & 10 & 11 & 10 & 10 \\
\hline \multirow[t]{5}{*}{ IX. } & 9.1 & 5 & 5 & 5 & 5 & 5 \\
\hline & 9.2 & 5 & 5 & 5 & 4 & 5 \\
\hline & 9.3 & 1 & 1 & 1 & 1 & 1 \\
\hline & 9.4 & 1 & 1 & 1 & 1 & 1 \\
\hline & Total & 12 & 12 & 12 & 11 & 12 \\
\hline \multirow[t]{3}{*}{ X. } & 10.1 & 4 & 4 & 5 & 4 & 5 \\
\hline & 10.2 & 4 & 5 & 4 & 4 & 5 \\
\hline & Total & 8 & 9 & 9 & 8 & 10 \\
\hline \multirow[t]{3}{*}{ XI. } & 11.1 & 4 & 3 & 4 & 3 & 4 \\
\hline & 11.2 & 4 & 5 & 5 & 4 & 4 \\
\hline & Total & 8 & 8 & 9 & 7 & 8 \\
\hline Porcentaje Total & & 139 & 136 & 140 & 133 & 143 \\
\hline
\end{tabular}

Tras obtener los resultados de la Tabla 17 se determinó que el apartado del Residente tiene mayor impacto con valor de usabilidad de "Bueno", puesto que de las 15 evaluaciones 8 obtuvieron esa característica, además de contar con mayor número de observaciones en las heurísticas VIII y IX, donde se apreció un menor número de usabilidad, en las cuales se dictaminaron mejoras. En la heurística VIII se estableció el reconocimiento de errores para el usuario, ya que la idea es ayudarlo más en la interacción, finalmente, en 
la heurística IX se estipuló identificar claramente los perfiles de usuario, toda vez que ocasionalmente se confundían los roles en el diseño. De esta forma se otorgó una oportunidad de mejora.

Conforme a los resultados de los diferentes perfiles del diseño global, se llegó a determinar que algunas partes necesitaban ser analizadas con mayor detalle, pues los usuarios confundían la interacción

Tabla 15. Valor de medición de porcentajes heurísticos y porcentaje total

\begin{tabular}{lccccc} 
Valor & Siglas & \multicolumn{2}{c}{ Porcentaje total por heurísticas } & Porcentaje total \\
& & 10 & 15 & 20 & 170 \\
\hline A) Muy malo & $\mathrm{MM}$ & $1-2$ & $1-3$ & $1-4$ & $1-34$ \\
\hline B) Malo & $\mathrm{M}$ & $3-4$ & $4-6$ & $5-8$ & $35-68$ \\
\hline C) Neutro & $\mathrm{N}$ & $5-6$ & $7-9$ & $9-12$ & $69-102$ \\
\hline D) Bueno & $\mathrm{B}$ & $7-8$ & $10-12$ & $13-16$ & $103-136$ \\
\hline E) Muy bueno & $\mathrm{MB}$ & $9-10$ & $13-15$ & $17-20$ & $137-170$ \\
\hline
\end{tabular}

Tabla 16. Porcentaje total de cinco evaluaciones al perfil Residente

\begin{tabular}{|c|c|c|c|c|c|c|}
\hline \multirow[t]{2}{*}{ Heurísticas } & \multirow[t]{2}{*}{$\begin{array}{l}\text { Porcentaje total por } \\
\text { Heurística }\end{array}$} & \multicolumn{5}{|c|}{ Residentes } \\
\hline & & 1 & 2 & 3 & 4 & 5 \\
\hline I & 10 & 9 & 9 & 9 & 9 & 10 \\
\hline II & 20 & 19 & 20 & 20 & 18 & 19 \\
\hline III & 15 & 13 & 11 & 14 & 13 & 14 \\
\hline IV & 20 & 17 & 17 & 15 & 18 & 18 \\
\hline $\mathrm{V}$ & 15 & 13 & 11 & 12 & 12 & 13 \\
\hline VI & 15 & 14 & 12 & 14 & 12 & 13 \\
\hline VII & 20 & 15 & 17 & 15 & 15 & 16 \\
\hline VIII & 15 & 11 & 10 & 11 & 10 & 10 \\
\hline IX & 20 & 12 & 12 & 12 & 11 & 12 \\
\hline$X$ & 10 & 8 & 9 & 9 & 8 & 10 \\
\hline XI & 10 & 8 & 8 & 9 & 7 & 8 \\
\hline Porcentaje Total & 170 & $\mathrm{MB} / 139$ & $\mathrm{~B} / 136$ & $\mathrm{MB} / 140$ & $\mathrm{~B} / 133$ & $\mathrm{MB} / 143$ \\
\hline
\end{tabular}

Tabla 17. Tabla de resultados finales por cada Perfil

\begin{tabular}{|c|c|c|c|c|c|c|c|}
\hline Evaluación & Residente & Coordinador & $\begin{array}{c}\text { Jefe del Depto. } \\
\text { Académico }\end{array}$ & $\begin{array}{l}\text { Integrante de } \\
\text { academia }\end{array}$ & $\begin{array}{c}\text { Asesor } \\
\text { Interno }\end{array}$ & $\begin{array}{l}\text { G.T. y } \\
\text { V. }\end{array}$ & S.E. \\
\hline & \multicolumn{7}{|c|}{ Número de evaluadores } \\
\hline & 15 & 6 & 6 & 15 & 15 & 1 & 6 \\
\hline 1 & 139 & 137 & 138 & 137 & 128 & 139 & 135 \\
\hline 2 & 136 & 133 & 137 & 142 & 130 & & 139 \\
\hline 3 & 140 & 136 & 134 & 144 & 130 & & 140 \\
\hline 4 & 133 & 135 & 135 & 135 & 130 & & 140 \\
\hline 5 & 143 & 135 & 136 & 142 & 136 & & 136 \\
\hline 6 & 134 & 137 & 136 & 142 & 136 & & 139 \\
\hline 7 & 102 & & & 136 & 133 & & \\
\hline 8 & 135 & & & 142 & 143 & & \\
\hline 9 & 135 & & & 133 & 134 & & \\
\hline 10 & 131 & & & 129 & 100 & & \\
\hline 11 & 135 & & & 129 & 124 & & \\
\hline 12 & 101 & & & 102 & 129 & & \\
\hline 13 & 137 & & & 133 & 135 & & \\
\hline 14 & 139 & & & 131 & 133 & & \\
\hline 15 & 135 & & & 128 & 136 & & \\
\hline
\end{tabular}


en algunas partes de la interfaz, por tal motivo no se contaba con un nivel estable de usabilidad. Tras la presentación del análisis realizado en la evaluación en la fase de los resultados de cada heurística, se adquirió una oportunidad de cómo minimizar o mitigar algunos problemas.

\subsection{Caso de estudio: Tutorías (T)}

\section{Identificación de los interesados que interactúan en el módulo (T)}

En el siguiente proceso de evaluación también se definieron los perfiles y acciones de los usuarios que intervienen en el módulo, los cuales se muestran en la Tabla 18.

Tabla 18. Listado de perfiles que intervienen en el módulo de "Tutorías"

\begin{tabular}{|c|c|}
\hline Perfil & Acciones \\
\hline Tutorado & $\begin{array}{l}\text { - Registra información que le sea requerida por el tutor para fines de integración del } \\
\text { trabajo. } \\
\text { - Realiza las actividades conjuntamente acordadas con el tutor para dar atención a sus } \\
\text { necesidades. } \\
\text { - Realiza evaluación institucional de la actividad tutorial. } \\
\text { - } \quad \text { Rena registro del Programa Institucional de Tutoría (PIT). } \\
\text { Realiza actividades de la acción tutorial. }\end{array}$ \\
\hline Tutor & 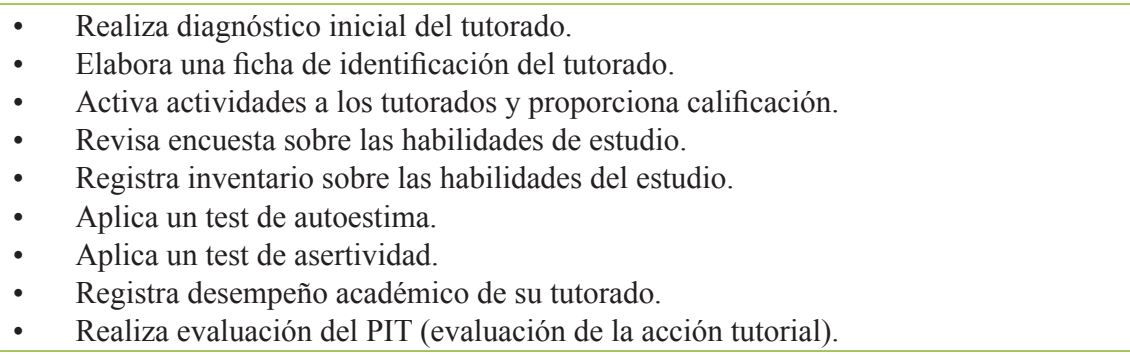 \\
\hline $\begin{array}{l}\text { Coordinador del } \\
\text { Departamento } \\
\text { académico }\end{array}$ & $\begin{array}{l}\text { - } \\
\text { - } \quad \text { Consigna tutores a los estudiantes. } \\
\text { - } \quad \text { Consulta relación de profesores que serán formados como tutores. } \\
\text { - } \quad \text { Consulta informe semestral. } \\
\text { - } \quad \text { Registra reportes semestrales. } \\
\text { - } \quad \text { Realiza cambios o reasignación de tutor. } \\
\text { - } \quad \text { Dansulta informe semestral del jefe del departamento académico. } \\
\text { - Genera informe semestral por carrera con los resultados obtenidos por carrera y las } \\
\text { metas establecidas en el Programa. }\end{array}$ \\
\hline $\begin{array}{l}\text { Jefe de Departamento } \\
\text { Académico }\end{array}$ & $\begin{array}{l}\text { - } \quad \text { Consulta diagnóstico institucional. } \\
\text { - } \quad \text { Registra docentes para ser Tutores. } \\
\text { - Genera Nombramiento de la acción tutorial. } \\
\text { - Genera un concentrado semestral de la acción tutorial con los estudiantes y tutores } \\
\text { del departamento académico correspondiente. } \\
\text { - } \quad \text { Gestiona por departamento académico el proceso de canalización. } \\
\text { - } \quad \text { Notifica al Coordinador del Programa, los resultados de la acción tutorial de su } \\
\text { departamento académico. } \\
\text { - } \quad \text { Gibera a los tutores. } \\
\text { Genera Carta de Liberación. }\end{array}$ \\
\hline $\begin{array}{l}\text { Coordinador } \\
\text { Institucional de Tutorías }\end{array}$ & $\begin{array}{l}\text { - } \quad \text { Consulta lista de tutores y tutorados correspondientes. } \\
\text { - } \quad \text { Consulta diagnóstico inicial. } \\
\text { - } \quad \text { Registra en que área se canaliza el tutorado. } \\
\text { - } \quad \text { Gensulta informe de acción tutorial. } \\
\end{array}$ \\
\hline
\end{tabular}


Tabla 19. Resultados detallados de cinco de las muestras de Evaluaciones Heurísticas al perfil Tutorado

\begin{tabular}{|c|c|c|c|c|c|c|}
\hline Heurísticas & Preguntas & Tutorados & & & & \\
\hline & & 1 & 2 & 3 & 4 & 5 \\
\hline \multirow[t]{3}{*}{ I } & 1.1 & 4 & 4 & 3 & 4 & 5 \\
\hline & 1.2 & 3 & 3 & 4 & 3 & 4 \\
\hline & Total & 7 & 7 & 7 & 7 & 9 \\
\hline \multirow[t]{5}{*}{ II } & 2.1 & 5 & 5 & 5 & 5 & 5 \\
\hline & 2.2 & 5 & 4 & 5 & 5 & 4 \\
\hline & 2.3 & 5 & 5 & 5 & 5 & 5 \\
\hline & 2.4 & 5 & 5 & 4 & 4 & 5 \\
\hline & Total & 20 & 19 & 19 & 19 & 19 \\
\hline \multirow[t]{4}{*}{ III } & 3.1 & 4 & 3 & 4 & 4 & 4 \\
\hline & 3.2 & 5 & 5 & 5 & 5 & 5 \\
\hline & 3.3 & 5 & 5 & 5 & 5 & 5 \\
\hline & Total & 14 & 13 & 14 & 14 & 14 \\
\hline \multirow[t]{5}{*}{ IV } & 4.1 & 5 & 4 & 5 & 5 & 5 \\
\hline & 4.2 & 3 & 4 & 5 & 4 & 4 \\
\hline & 4.3 & 5 & 5 & 5 & 5 & 5 \\
\hline & 4.4 & 3 & 3 & 3 & 3 & 3 \\
\hline & Total & 16 & 16 & 18 & 17 & 17 \\
\hline \multirow[t]{4}{*}{$\mathrm{V}$} & 5.1 & 2 & 2 & 2 & 3 & 3 \\
\hline & 5.2 & 3 & 3 & 4 & 4 & 4 \\
\hline & 5.3 & 4 & 4 & 4 & 4 & 4 \\
\hline & Total & 9 & 9 & 10 & 11 & 11 \\
\hline \multirow[t]{4}{*}{ VI } & 6.1 & 5 & 5 & 5 & 5 & 5 \\
\hline & 6.2 & 4 & 5 & 5 & 4 & 5 \\
\hline & 6.3 & 5 & 5 & 5 & 5 & 5 \\
\hline & Total & 14 & 15 & 15 & 14 & 15 \\
\hline \multirow[t]{5}{*}{ VII } & 7.1 & 5 & 5 & 5 & 5 & 5 \\
\hline & 7.2 & 4 & 4 & 5 & 5 & 5 \\
\hline & 7.3 & 5 & 5 & 5 & 4 & 5 \\
\hline & 7.4 & 5 & 5 & 5 & 5 & 5 \\
\hline & Total & 19 & 19 & 20 & 19 & 20 \\
\hline \multirow[t]{4}{*}{ VIII } & 8.1 & 3 & 4 & 4 & 3 & 4 \\
\hline & 8.2 & 4 & 4 & 4 & 4 & 4 \\
\hline & 8.3 & 5 & 5 & 5 & 5 & 5 \\
\hline & Total & 12 & 13 & 13 & 12 & 13 \\
\hline \multirow[t]{5}{*}{ IX } & 9.1 & 5 & 5 & 5 & 5 & 5 \\
\hline & 9.2 & 4 & 4 & 5 & 4 & 5 \\
\hline & 9.3 & 1 & 1 & 1 & 1 & 1 \\
\hline & 9.4 & 4 & 4 & 4 & 4 & 4 \\
\hline & Total & 14 & 14 & 15 & 14 & 15 \\
\hline \multirow[t]{3}{*}{$X$} & 10.1 & 5 & 5 & 5 & 5 & 5 \\
\hline & 10.2 & 4 & 4 & 4 & 4 & 4 \\
\hline & Total & 9 & 9 & 9 & 9 & 9 \\
\hline \multirow[t]{3}{*}{ XI } & 11.1 & 4 & 4 & 4 & 4 & 4 \\
\hline & 11.2 & 4 & 4 & 4 & 4 & 4 \\
\hline & Total & 8 & 8 & 8 & 8 & 8 \\
\hline $\begin{array}{l}\text { Porcentaje } \\
\text { Total }\end{array}$ & & 142 & 142 & 148 & 144 & 150 \\
\hline
\end{tabular}

Aplicación y resultados de la evaluación a un perfil

En la evaluación del módulo de "Tutorías" se tomó una muestra de quince personas del perfil Tutorado. En la Tabla 19 son presentados los resultados Heurísticos obtenidos de cinco de las muestras, en ella se 
visualizan los totales de cada Heurística y el total de cada Evaluación. Una vez obtenidos los resultados de las cinco muestras del perfil tutorado se observó que existía semejanza en los valores totales de la puntuación, lo que permitió seguir aplicando evaluaciones con la finalidad de mejorar la eficiencia de los resultados. Los formatos utilizados para la evaluación se aplicaron para todos los perfiles que se mencionan en la Tabla 18.

Tras los resultados obtenidos se elaboró una tabla de medición que otorga valor al total de cada principio heurístico y al porcentaje total de la evaluación, obteniendo como resultado el nivel de usabilidad del diseño (ver Tabla 20).

Tabla 20. Valor de medición de porcentajes heurísticos y porcentaje total

\begin{tabular}{lccccc}
\multicolumn{1}{c}{ Valor } & Siglas & \multicolumn{2}{c}{ Porcentaje total por heurísticas } & Porcentaje total \\
& & 10 & 15 & 20 & 170 \\
\hline F) Muy malo & MM & $1-2$ & $1-3$ & $1-4$ & $1-34$ \\
\hline G) Malo & $\mathrm{M}$ & $3-4$ & $4-6$ & $5-8$ & $35-68$ \\
\hline H) Neutro & $\mathrm{N}$ & $5-6$ & $7-9$ & $9-12$ & $69-102$ \\
\hline I) Bueno & $\mathrm{B}$ & $7-8$ & $10-12$ & $13-16$ & $103-136$ \\
\hline J) Muy bueno & $\mathrm{MB}$ & $9-10$ & $13-15$ & $17-20$ & $137-170$ \\
\hline
\end{tabular}

Con respecto a los resultados detallados de la Tabla 19 y la utilización de los valores de medición mencionados previamente, en la Tabla 21 es presentado el porcentaje total obtenido de la muestra de cinco evaluaciones al perfil Tutorado, dando como resultado cinco evaluaciones con valor "Muy bueno", lo cual demuestra que el diseño contiene niveles de usabilidad. Puesto que los resultados de cada heurística fueron diferentes se identificaron aquellas que tuvieron bajo puntaje y se procedió a realizar un análisis de usabilidad para detectar problemas y proponer mejoras que faciliten al usuario el manejo del diseño.

Tabla 21. Porcentaje total de cinco evaluaciones al perfil Tutorado

\begin{tabular}{ccccccc} 
Heurísticas & $\begin{array}{c}\text { Porcentaje total por } \\
\text { Heurística }\end{array}$ & $\mathbf{1}$ & $\mathbf{2}$ & $\mathbf{3}$ & $\mathbf{4}$ & $\mathbf{5}$ \\
I & 10 & 7 & 7 & 7 & 7 & 9 \\
\hline II & 20 & 20 & 19 & 19 & 19 & 19 \\
\hline III & 15 & 14 & 13 & 14 & 14 & 14 \\
\hline IV & 20 & 16 & 16 & 18 & 17 & 17 \\
\hline V & 15 & 9 & 9 & 10 & 11 & 11 \\
\hline VI & 15 & 14 & 15 & 15 & 14 & 15 \\
\hline VII & 20 & 19 & 19 & 20 & 19 & 20 \\
\hline VIII & 15 & 12 & 13 & 13 & 12 & 13 \\
\hline IX & 20 & 9 & 9 & 14 & 14 & 15 \\
\hline X & 10 & 8 & 8 & 8 & 8 & 8 \\
\hline XI & 10 & $\mathbf{M B} / \mathbf{1 4 2}$ & $\mathbf{M B} / \mathbf{1 4 2}$ & $\mathbf{M B} / \mathbf{1 4 8}$ & $\mathbf{M B} / \mathbf{1 4 4}$ & $\mathbf{M B} / \mathbf{1 5 0}$ \\
\hline Porcentaje Total & $\mathbf{1 7 0}$ & & & & &
\end{tabular}

\section{Obtención de resultados generales}

En la Tabla 22 se visualiza el número de evaluadores de cada perfil, mostrando los porcentajes finales obtenidos. Con la utilización de la Tabla 20 del apartado (Porcentaje Total) se puede identificar el nivel de Usabilidad obtenido por cada Evaluación.

A partir de los resultados obtenidos de la evaluación de 15 de los evaluadores del perfil Tutorado de la Tabla 22, se obtuvo como nivel de usabilidad con mayor puntuación el valor "Bueno", lo que significa que no existe una satisfacción total, por lo que se propuso la implementación de nuevas mejoras a las heurísticas que tuvieron menos puntuación:

I. Visibilidad del diseño del prototipo: en esta heurística se observó que algunos usuarios no identificaban claramente en qué apartado del sistema se encontraban debido a la similitud entre plantillas, por lo que se mejoró el diseño del sistema para que el usuario pueda identificar en dónde se encuentra y hacer más notables los enlaces para mejorar su visualización. 
Tabla 22. Tabla de resultados finales por cada Perfil

\begin{tabular}{cccccc} 
Evaluación & Tutorado & Tutor & $\begin{array}{c}\text { Coordinador del } \\
\text { Departamento } \\
\text { Académico } \\
\text { Número de evaluadores }\end{array}$ & $\begin{array}{c}\text { Jefe del } \\
\text { Departamento } \\
\text { Académico }\end{array}$ & $\begin{array}{c}\text { Coordinador } \\
\text { Institucional de } \\
\text { Tutorías }\end{array}$ \\
\hline 1 & 15 & 15 & 6 & 6 & 132 \\
\hline 2 & 142 & 101 & 126 & 127 & 132 \\
\hline 3 & 142 & 102 & 130 & 136 & \\
\hline 4 & 148 & 139 & 99 & 135 & \\
\hline 5 & 144 & 132 & 133 & \\
\hline 6 & 150 & 134 & 127 & \\
\hline 7 & 133 & 138 & & & \\
\hline 8 & 130 & 130 & & & \\
\hline 10 & 132 & 131 & & & \\
\hline 11 & 132 & 137 & & & \\
\hline 12 & 100 & 133 & & & \\
\hline 13 & 136 & 103 & & & \\
\hline 14 & 133 & 130 & & & \\
\hline 15 & 131 & 131 & & & \\
\hline
\end{tabular}

V. Prevención de errores: algunos de los usuarios cometían errores al momento de ingresar los datos en el sistema, por lo que se agregaron guías a cada campo donde se ingresa la información, así como el agregado de mensajes de prevención de errores, logrando que el sistema no induzca a cometer errores.

IX. Tratamiento del contenido: algunos de los evaluadores observaron que los perfiles eran parecidos y ocasionaba confusión al momento de realizar la evaluación, por lo que se resaltaron las preferencias de cada usuario.

Con los resultados obtenidos de los demás perfiles se determinó mejorar la experiencia del usuario en aquellas partes que necesitaban ser analizadas con mayor detalle, de modo que permita lograr el grado de satisfacción ofrecido en las distintas secciones y elementos del diseño en cuanto al cumplimiento de las tareas u objetivos para las que fue diseñada, evitando la confusión y conflicto de interacción de diseño.

\section{Conclusiones}

Este artículo ha presentado el uso de un método de Evaluación Heurística de Nielsen enfocado en la usabilidad, evaluando el diseño de interfaz de los módulos denominados "Residencias Profesionales" y "Tutorías". Las características de los dos casos de estudio propiciaron un entorno adecuado para la evaluación mediante el empleo de los principios heurísticos, permitiendo identificar la subjetividad adherida a los atributos de usabilidad en el diseño, así como la detección del origen de los problemas, brindando recomendaciones para que estos puedan ser corregidos en las fases tempranas del diseño (Holzinger, 2005).

Los resultados obtenidos en el primer caso de estudio del módulo de "Residencias Profesionales" derivaron de los diferentes perfiles y el número de evaluadores que se tomaron como muestra. En la Tabla 23 se registraron los resultados de las evaluaciones con valores: Muy bueno, Bueno, Neutro, Malo y Muy malo, mostrando el porcentaje de usabilidad de cada perfil en el diseño. Se obtuvo como resultado mayor, con la cantidad de 39, el valor "Bueno", seguidamente 21 "Muy bueno", y finalmente 4 "Neutro" en todo el diseño.

Además de obtener los resultados del diseño de la interfaz de cada perfil, se identificaron algunos puntos de mejora en aspectos referentes a la interacción del usuario; por ejemplo, se visualizó que algunos de los evaluados tenían diferentes capacidades de interpretación referente al significado de las palabras e iconos utilizados en el diseño, así como la falta de análisis en acciones a realizar, ya que cometían errores que los llevarían a la pérdida de información.

En cuanto al diseño de la interfaz se detectó en algunos casos la falta de mensajes que ayudaran al usuario a verificar si era la acción que deseaba realizar. Otro punto que se manifestó fue el tener el mismo diseño para todos los perfiles y que en ocasiones algunos podían ser dos roles al mismo tiempo. Al llegar a interactuar con las dos interfaces se confundía la sesión en la que se encontraban, por lo cual se propuso 
Tabla 23. Tabla de resultados de usabilidad del módulo de "Residencias Profesionales"

\begin{tabular}{lccccc}
\multicolumn{1}{c}{ Perfil } & Número de Evaluadores & \multicolumn{4}{c}{ Valor de Usabilidad } \\
Residente & 15 & 5 & 8 & 2 & Muy malo \\
\hline Coordinador & 6 & 2 & 4 & & \\
\hline Jefe del Depto. Académico & 6 & 2 & 4 & & \\
\hline Integrante de academia & 15 & 6 & 8 & 1 \\
\hline Asesor Interno & 15 & 1 & 13 & 1 \\
\hline G.T. y V. & 1 & 1 & & & \\
\hline S.E. & 6 & 4 & 2 & & \\
\hline
\end{tabular}

que en cada menú de los diferentes roles se determinara para quién era dirigido, por ejemplo, Asesor o Revisor. Tras estos y otros puntos identificados tanto en el diseño como en la interacción del usuario, se logró determinar que se pueden seguir implementando mejoras en los puntos frágiles del proyecto para posteriormente tener una elaboración factible del sistema.

Los resultados obtenidos en el segundo caso de estudio del módulo de "Tutorías" derivaron de los diferentes perfiles y el número de evaluadores que se tomaron como muestra. En la Tabla 24 se registraron los resultados de las evaluaciones con valores: Muy bueno, Bueno, Neutro, Malo y Muy malo, mostrando el porcentaje de usabilidad de cada perfil en el diseño; se obtuvo como resultado mayor, con la cantidad de 28, el valor "Bueno", seguidamente 9 "Muy bueno" y por último 6 "Neutro" en todo el diseño.

Tabla 24. Tabla de resultados de usabilidad del módulo de "Tutorías"

\begin{tabular}{|c|c|c|c|c|c|c|}
\hline \multirow[b]{2}{*}{ Perfil } & \multirow{2}{*}{$\begin{array}{l}\text { Número de } \\
\text { Evaluadores }\end{array}$} & \multicolumn{5}{|c|}{ Valor de Usabilidad } \\
\hline & & $\begin{array}{l}\text { Muy } \\
\text { bueno }\end{array}$ & Bueno & Neutro & Malo & $\begin{array}{l}\text { Muy } \\
\text { malo }\end{array}$ \\
\hline Tutorado & 15 & 5 & 8 & 2 & & \\
\hline Tutor & 15 & 4 & 9 & 2 & & \\
\hline $\begin{array}{l}\text { Coordinador del Departamento } \\
\text { Académico }\end{array}$ & 6 & & 5 & 1 & & \\
\hline $\begin{array}{l}\text { Jefe del Departamento } \\
\text { Académico }\end{array}$ & 6 & & 5 & 1 & & \\
\hline $\begin{array}{l}\text { Coordinador Institucional de } \\
\text { Tutorías }\end{array}$ & 1 & & 1 & & & \\
\hline
\end{tabular}

Esta evaluación motivó al objetivo del presente artículo para comprender los problemas por resolver, ya que se encontraron aspectos referentes a la interacción del usuario, es decir, que los evaluadores al momento de proporcionar la información en los campos correspondientes no tenían guías de ayuda para saber qué datos ingresar, lo que ocasionaba que cometieran errores, por lo que se propuso agregar guías de ayuda como mejora del diseño.

Se encontró que algunos de los evaluados no podían leer el texto fácilmente, puesto que la tipografía que se utilizaba no tenía un tamaño de texto apropiado para los usuarios, por lo cual se propuso utilizar una fuente de texto adecuada, además de tener un interlineado y separación de palabras suficiente, de tal manera que pueda ser leído. Estas propuestas de mejora en el diseño permitirán posteriormente tener un desarrollo factible del sistema.

Entre los aspectos a destacar, como resultado de este trabajo, mediante los once principios heurísticos de Nielsen se identificó que los usuarios prefieren un diseño agradable, involucrando en este aspecto la disposición de elementos sin sobrecarga de imágenes o de información, búsquedas de resultados apropiados o precisos y que durante el recorrido entre los bocetos de diseño sea sencillo y fácil de recordar, con el fin de obtener la satisfacción y potenciación de la participación del usuario.

Al mismo tiempo se obtuvieron evaluaciones prácticas y sencillas, sin embargo, no solo se llegó a medir la usabilidad en el contenido, sino también la usabilidad en la experiencia del usuario, basado en tres aspectos: imagen en el diseño interactivo, buen contenido y la obtención de respuestas acertadas en la interacción del prototipo. 
Es relevante que un diseño debe estar enfocado en los usuarios que llegarán a interactuar con la interfaz. Con los diversos resultados obtenidos en los dos casos de estudio se estableció que los diseñadores no tomaron en cuenta los diferentes tipos de usuarios respecto a un perfil, algunos no tenían la misma capacidad de comprender la interacción, impidiendo lograr la satisfacción, así mismo, provocando que los usuarios desertaran en el manejo de la interfaz, por lo cual se les manifestó a los diseñadores investigar y analizar los diversos usuarios que puede llegar a tener el sistema.

Se discutió sobre el idéntico diseño para todos los perfiles, toda vez que en el caso de los menús no todos contaban con el nombre del perfil correspondiente, lo que ocasionó confusión al momento de interactuar con las dos interfaces, por lo que se planteó al diseñador determinar un nombrado en la parte superior de cada menú. De igual forma se pudo percibir que los evaluadores no concordaban con la tipografía utilizada en el diseño ya que en distintitas ocasiones los usuarios tenían que acercarse a la pantalla para poder leer el contenido, por lo que se expresó al diseñador la integración de una mejor fuente de texto en los diversos apartados de los módulos.

Otro factor importante que se consideró en la evaluación es que los diseñadores integraron en algunas partes vocabulario no conocido por parte de los usuarios. Al estar navegando se preguntaban a qué se referían algunas palabras como "Módulo", con lo cual no sabían si seguir con la interacción o asociarlo con otro aspecto que consideraban referente a la palabra, de esta forma en algunas ocasiones tenían la incertidumbre de si era correcta su definición o esperar a preguntar a otro usuario, lo que conllevó a analizar esta característica del diseño, por lo cual se determinó una mejora para que el diseñador utilice vocabulario referente a los usuarios.

Tras estos sucesos fue necesario resaltar la información de interés de cada usuario, por lo que se presentaron las retroalimentaciones a los diseñadores para poder llegar a tener una mejor usabilidad en el diseño de la interfaz en los dos casos de estudio. En este sentido, es indispensable elaborar un proceso de evaluación de usabilidad temprana en la fase de diseño, otorgando posteriormente en las siguientes fases un grado de factible en el desarrollo del sistema. Por lo tanto, esta evaluación puede ser empleada en cualquier diseño de interfaz web, dirigido por un prototipo de navegación. Finalmente, se enfatiza que una Evaluación Heurística consta de diferentes pasos siendo cada uno de ellos crucial para lograr el éxito.

\section{Declaración de conflicto de intereses}

Los autores declaran no tener conflicto de intereses con respecto a la investigación, autoría y/o publicación de este artículo.

\section{Referencias}

Abran, A., Khelifi, A., Suryn, W., \& Seffah, A. (2003). Consolidating the ISO Usability Models. In Proceedings of 11 th international software quality management conference (pp. 23-25). Glasgow, Scotland, UK.

Bevan, N. (1991). What is usability. In Human Aspects in Computing: Design and Use of Interactive Systems and Work With Terminals.

Blackmon, M. H., \& Bainbridge, W. S. (2004). Cognitive walkthrough. In Encyclopedia of human-computer interaction (pp. 104-107).

Chimarro Chipantiza, V. L., Mazón Olivo, B. E., \& Cartuche Calva, J. J. (2015). La usabilidad en el desarrollo de software. Universidad Técnica de Machala. Retrieved from http://repositorio.utmachala.edu.ec/ handle/48000/6878

Claros Gómez, I. D. (2006). Lineamientos de Diseño para el Desarrollo de Aplicaciones Usables bajo Entornos Web. Retrieved September 26, 2018, from http://artemisa.unicauca.edu.co/ iclaros/usabilidad/tecnicas.htm

Holzinger, A. (2005). Usability engineering methods for software developers. Communications of the ACM, 48(1), $71-74$.

International Organization for Standardization. (1998). ISO 9241-11:1998 Ergonomic requirements for office work with visual display terminals (VDTs) -- Part 11: Guidance on usability. Retrieved from https://www.sis.se/ api/document/preview/611299/

Lorés, J., \& Granollers, T. (2004). La Ingeniería de la Usabilidad y de la Accesibilidad aplicada al diseño y desarrollo de sitios web. Lleida. 
Manzari, L., \& Trinidad-Christensen, J. (2006). User-Centered Design of a Web Site for Library And Information Science Students: Heuristic Evaluation And Usability Testing. Information Technology and Libraries, 25(3), 163-169. https://doi.org/10.6017/ital.v25i3.3348

Montes de Oca, A. (2004). Arquitectura de información y usabilidad: nociones básicas para los profesionales de la información. Acimed, 12(6).

Nielsen, J. (1994a). 10 Usability Heuristics for User Interface Design. Retrieved October 1, 2018, from https://www. nngroup.com/articles/ten-usability-heuristics/

Nielsen, J. (1994b). Usability Engineering. San Francisco, CA.: Morgan Kaufmann Publishers Inc. Retrieved from https://dl.acm.org/citation.cfm?id=2821575

Nielsen, J. (1994c). Usability inspection methods. In Conference companion on Human factors in computing systems (pp. 413-414).

Nielsen, J. (1995). How to conduct a heuristic evaluation. Retrieved from http://www.ingenieriasimple.com/ usabilidad/HeuristicEvaluation.pdf

Preece, J., Rogers, Y., \& Sharp, H. (2005). Design de interação: além da interação homem-computador.

Reyes Vera, J. M., \& Libreros Giraldo, F. A. (2011). Método para la evaluación integral de la usabilidad en sistemas e-Learning. Educación En Ingeniería, 6(12), 69-79. Retrieved from https://www.educacioneningenieria. org/index.php/edi/article/view/126

Sánchez I., J. (2000). “Evaluación de Usabilidad de Sitios Web”: Método de evaluación heurística. Retrieved from http://bolivarzuiga.com/wp-content/uploads/2009/11/pautaevaluacionheuristica.pdf

Suárez Torrente, M. del C. (2011). Sirius: sistema de evaluación de la usabilidad web orientado al usuario y basado en la determinación de tareas críticas. Universidad de Oviedo.

Wharton, C. (1994). The cognitive walkthrough method: A practitioner's guide. In Usability inspection methods.

\section{Sobre los autores}

\section{Guadalupe García Toribio}

Ingeniera en Informática. Instituto Tecnológico Superior de Teziutlán, 2016. Actualmente estudiante de Maestría en Sistemas Computacionales del Instituto Tecnológico de Apizaco. Su investigación está relacionada con el desarrollo e implementación del módulo de "Tutorías" de una Institución de Educación Superior.

\section{Yesenia Polvo Saldaña}

Ingeniera en Tecnologías de la Información y Comunicaciones. Instituto Tecnológico de Apizaco, 2016. Actualmente estudiante de Maestría en Sistemas Computacionales del Instituto Tecnológico de Apizaco. $\mathrm{Su}$ investigación está relacionada con el desarrollo e implementación del módulo de "Residencias Profesionales" de una Institución de Educación Superior.

\section{José Juan Hernández Mora}

Ingeniero en Computación, Universidad Autónoma de Tlaxcala, 1994. Maestro en Ciencias Computacionales por el Centro Nacional de Investigación y Desarrollo Tecnológico del TecNM, 2003. Profesor Investigador del Tecnológico de Apizaco del TecNM. Docente de la Maestría en Sistemas Computacionales del Instituto Tecnológico de Apizaco.

\section{María Janaí Sánchez Hernández}

Licenciada en Informática, Instituto Tecnológico de Apizaco, 2001. Maestra en Ciencias de la Computación, Instituto Tecnológico de Apizaco, 2006. Docente del Departamento de Sistemas y Computación, 2006. Coordinadora de la Maestría en Sistemas Computacionales, del Instituto Tecnológico de Apizaco, 2015. 


\section{Higinio Nava Bautista}

Ingeniero en Computación, Universidad Autónoma de Tlaxcala, 2007. Maestro en desarrollo de Software, Instituto Universitario en Tecnologías y Humanidades, 2012. Docente en la Carrera de Ingeniería en Tecnologías de la Información y Comunicaciones. Docente de la Maestría en Sistemas Computacionales del Instituto Tecnológico de Apizaco.

\section{César Alberto Collazos Ordóñez}

Ingeniero en Sistemas y Computación, Universidad de los Andes, 1993. Doctor en Ciencias Mención Computación, Universidad de Chile, 2004. Estancias postdoctorales en el Grupo CARL (Collaborative Applications Research Laboratory) de la Universidad de Chile, 2004 y en el Grupo C.H.I.C.O (Computer Human Interaction and Collaboration) de la Universidad Castilla-La Mancha, España, 2005. Vicepresidente de la Sociedad Colombiana de Computación. Coordinador Grupo IDIS (Investigación y Desarrollo en Ingeniería del Software), Universidad del Cauca.

\section{Julio Ariel Hurtado Alegría}

Ingeniero en Electrónica y Telecomunicaciones, Universidad del Cauca, 1997. Especialista en Redes y Servicios Telemáticos, Universidad del Cauca. Especialista en Procesos para el Desarrollo de Software, Universidad San Buenaventura, 2002. Doctor en Ciencias de la Computación de la Universidad de Chile, 2012. Profesor Titular de la Universidad del Cauca. Miembro del Grupo IDIS desde el año 2005. 